\title{
4 \\ RESEÑA DE AVANCES Y EXPLORACIONES INICIALES (PROPÓSITO, DISEÑO, DIAGRAMACIÓN)*
}

"Quedar cautivado por los componentes culturales específicos,
estudiar con atención los procesos de raciocinio,
caer de bruces sobre los sistemas simbólicos...
Todo eso no hace desaparecer al mundo;
al contrario, lo expone a la vista"

Clifford Geertz

La interpretación de las culturas 
Advierto con frecuencia las significativas dificultades que se le plantean, al estudiante de grado y posgrado, a la hora de tener que producir comunicaciones escritas de corte académico o científico. Entre otras, más o menos comunes y habituales: insuficiencias en el manejo de las referencias conceptuales, rupturas de la cohesión textual en la redacción de textos informativos y, aún, en la coherencia lógica y argumentativa de sus prácticas de escritura, etc. ${ }^{1}$

Considerando que el cuadro de situación se presenta como demasiado complejo y diversificado, propongo en cambio retrabajar aquí sólo cuestiones vinculadas con la textualización de los procesos de investigación y la comunicación de sus resultados. La puesta en texto de lo antes adquirido, una instancia singular de la actividad investigativa, por cierto, que -además de vincularse con los recorridos de narratividad que interesan al tramo actual del Proyecto de Semiótica y Análisis del Discurso que dirijo- se constituye en el fundamento básico de la difusión del avance y el acrecentamiento del conocimiento científico: competencias cognitivas esenciales cuya progresiva adquisición, en el desarrollo de nuestras prácticas didácticas, no debieran descuidarse, ni tampoco, solaparse.

Dichas dificultades de textualización limitan o condicionan (en el caso particular de los investigadores en formación) la generación escritural, retórica y estilística de los textos que producen. Suelen transformarse, además, en serios obstáculos o impedimentos para la plasmación escrita de un conjunto de ideas y razones, que logren conformar a la vez un discurso argumentativo válido, característico de la ciencia y la investigación (aunque sólo sea una práctica básica, inicial o incipiente; lo cual no debiera implicar afectación o perjuicio de su necesaria solidez, fundamentación, articulación, a la vez sintética y sincrética).

En otras palabras: en vez de reflexionar aquí acerca de las dificultades e inconvenientes que entraña la configuración textual, discursiva y narrativa de

\footnotetext{
${ }^{1}$ Dejo obviamente de lado las numerosísimas cuestiones normativas que en general se presentan al elaborar este tipo de informes escritos: falencias esenciales, tanto en el dominio ortográfico, como en lo sintáctico y lo léxico, así como en el empleo de las reglas de acentuación y puntuación, las que demandarían implementar otro tipo de "soluciones".
} 
toda información argumentativa o tematización académica, me ocuparé del problema pero desde un "atajo" (más) pragmático y empírico, relacionado con la culminación de un ejercicio investigativo. Esto es, sugiriendo modos concretos, puntuales de presentar los resultados del esfuerzo llevado a cabo, desde su concepción inicial hasta su temporaria y siempre provisoria conclusión.

Propongo a continuación entonces un diseño -diagrama, formato, ejemplificación-que quisiera nomenclarse como provisorio o experimental modelo de informe de investigación en proceso, dadas sus características particulares, las cuales lo singularizan y diferencian de los demás "tipos" habituales de informes de investigación. Al respecto se procurarán integrar o reunir condiciones e ítems propios, más o menos estandarizados, de toda redacción de proyectos de investigación ${ }^{2}$, con otros registros particulares o específicos de ciertos informes de avance, producidos en correlación con "estudios exploratorios"3 ${ }^{3}$ aplicados a diferentes objetos temáticos.

\footnotetext{
${ }^{2}$ Cf. por ejemplo los documentos de trabajo expuestos en De signos y sentidos, 3. Construcción de proyectos en investigaciones aplicadas (2006), en esta misma serie de publicaciones periódicas.

${ }^{3}$ Las indagaciones exploratorias habitualmente permiten adquirir cierto grado de familiaridad con el fenómeno u objeto a investigar, especialmente en el caso de los investigadores en formación. A la vez, aunque en un determinado estudio predominen condiciones o características esencialmente exploratorias, el mismo puede además contener o anticipar elementos propios de otros tipos de investigaciones: descriptivas (que señalan y especifican propiedades o variables del fenómeno), predictivas (cuando predicen posibilidades o validaciones incipientes), explicativas (si delimitan y fundamentan causales de los eventos, procesos o acontecimientos analizados), etc. Procurando integrar diversas características de la práctica investigativa y la información de sus resultados, esta propuesta de trabajo quisiera contribuir, en términos más o menos inmediatos a la suplencia de las carencias detectadas durante la coordinación de numerosos seminarios de grado y posgrado, y de algún modo dar respuesta a determinadas inquietudes y expectativas de mejoramiento didáctico.
} 


\section{DIAGRAMA DE DISEÑO DEL CONTENIDO DEL INFORME ${ }^{4}$ \\ (UNA PROPUESTA MODULAR)}

\section{PARATEXTOS}

\subsection{PORTADA}

Entre otros datos se podrían incluir, por ejemplo, (a) el título de la investigación (eventualmente: un subtítulo y/o epígrafe) ${ }^{5}$; (b) autor(es) del informe; (c) institución académica (cátedra o seminario, si correspondiera); (d) lugar y fecha de la presentación.

\section{2. ÍNDICE O SUMARIO}

Esquema analítico del contenido, discriminado por ítems (apartados, subapartados) e indicación de las páginas correspondientes.

\section{INTRODUCCIÓN}

La introducción del trabajo debiera constar de, por lo menos, tres aspectos básicos: (a) la presentación (resumen) del informe, (b) algunas precisiones

\footnotetext{
${ }^{4}$ La elaboración de un informe (exploratorio o de avances en la investigación, como en este caso) permite "representar" la síntesis, es decir "la culminación del ejercicio investigador, en forma de documento para su lectura y evaluación. Este documento puede adoptar distintas formas, de conformidad con las características del público al que principalmente se dirige (profano, experto), del medio empleado para su divulgación (monografía, revista, comunicación de congreso, informe técnico), y del ingenio y estilo comunicativo del investigador, fundamentalmente" (Forni, Kreimer y Thomas, s/d)

${ }^{5}$ El título (y, según los casos, el agregado de un subtítulo e incluso un texto seleccionado como epígrafe) debiera(n) explicitar lo más claramente posible el tema y el objeto de la investigación ya que, a modo de presentación y de herramienta descriptiva inicial, permite(n) dar cuenta y ubicar rápidamente al lector acerca de aquello que se está investigando y anticipar brevemente la índole específica del informe presentado.
} 
acerca de la propuesta investigativa general, y (c) ubicación (del informe) en el marco de dicho proyecto ${ }^{6}$.

\subsection{PRELIMINARES}

En este apartado podrían presentarse o anticiparse, en forma de abstract o resumen, las características generales del siguiente informe o comunicación escrita ${ }^{7}$.

\subsection{ARGUMENTOS}

Este ítem funciona como encabezamiento sintético -tanto como fuera posibledel informe de avance, ya que sitúa los principales elementos o argumentos centrales del proyecto general en el cual se inscriben: por ejemplo (a) el tema, problema, objeto e impacto ${ }^{8}$ de la investigación; (b) pueden además incluirse (sería muy importante hacerlo) la situación motivadora, algunos de los interrogantes o preguntas ${ }^{9}$ que se intentarán responder, (pre)hipótesis iniciales de trabajo, presupuestos y alcances de la propuesta, síntesis del estado del arte sobre la cuestión por indagar, breves indicaciones acerca del marco teórico o "modelo" a partir del cual se desarrolla la investigación, etc.

\footnotetext{
${ }^{6}$ Esta sección introductoria del informe está destinada a ofrecer una visión global de la investigación en curso, y a destacar su relevancia para el conocimiento (¿o el desarrollo? ¿o la solución?) del problema y/o el objeto de análisis investigativo. En ella, el/la informante define, caracteriza y contextualiza los aspectos que se desarrollarán a continuación. 7 Teniendo en cuenta la diversidad de lectores posibles, resultará conveniente redactar todo el informe con un lenguaje claro y sencillo, relativamente "neutro" y "objetivo" (sintaxis simple, estructuración precisa, oraciones cortas y redacción lineal, etc.).

${ }^{8}$ Un problema es aquello susceptible de ser efectivamente investigado de un modo sistemático, y no cualquier cuestión, de entre las muchas que componen una agenda posible de temas de investigación. Proponerse objetivos y formularse adecuadas preguntas relativas a ellos, desarrollar una adecuada justificación de los mismos y demostrar su factibilidad (posibilidades reales de abordarlo con éxito) son algunos de los pasos necesarios para definir un problema de investigación, instancias previas a la construcción de su objeto y a la determinación de su impacto (significación, relevancia, eventuales aprovechamientos, posibilidades de transferencia, etc.).

${ }^{9}$ Una minuciosa formulación de preguntas e interrogantes significativos, en cierto modo ponen a prueba la originalidad, interés, pertinencia, alcance, consistencia, etc. del problema elegido y los pasos seleccionados para abordarlo (para la elección de un marco teórico y metodológico adecuado, por ejemplo).
} 


\subsection{CONTEXTO}

En la reseña del contexto de investigación se sintetizan dificultades, soluciones, actividades previstas, etc. en relación con lo expresado en el punto anterior; y se da cuenta del estado transicional, siempre provisorio del desarrollo de la cuestión o del problema investigativo, a propósito de los encuadres referenciales del proyecto que ya fueran mencionados (marco teórico y analítico, incluidos), el corpus textual-operacional tentativo, etc., y del recorte producido para la elaboración de este informe específico.

\subsection{AGREGADOS}

Otras precisiones, en caso de considerárselas necesarias.

\section{DESARROLLO}

La parte medular del trabajo (a) dará cuenta de las indagaciones efectuadas (en relación con las pautas y previsiones establecidas en el diseño del proyecto general, según lo que ya ha sido informado en la "introducción" del reporte) y (b) explicitará los recursos, técnicas e instrumentos teóricos, metodológicos, etc. que fueron seleccionados para llevar a cabo dicha investigación preliminar (suponiendo que se tratara de un estudio exploratorio).

\subsection{ESTRATEGIAS}

Se resumirán las alternativas y opciones de la perspectiva metodológica ${ }^{10}$ adoptada y la(s) estrategia(s) particular(es) de investigación que se pondrá(n)

\footnotetext{
${ }^{10}$ Las metodologías de investigación en ciencias humanas y sociales suelen agruparse en dos perspectivas (casi) antagónicas: metodologías cualitativas ("humanistas") y cuantitativas ("cientificistas"). Las primeras priorizan el análisis de lo individual y concreto -lo descriptivo, lo lingüístico, los aspectos 'micro' de la vida social-y operan a partir de la comprensión e interpretación de los significados intersubjetivos; las segundas, en cambio, enfatizan la explicación, la contrastación empírica y la medición "objetiva" de los fenómenos sociales, y postulan la validez de "el método científico" (de las exactas y naturales), el principio de causalidad, la formulación de leyes generales en el análisis de lo social, etc. Se asocian las primeras con el denominado contexto de descubrimiento (con la
} 
en práctica o que se ejemplificarán a continuación: es decir, se reseñarán las tareas "operacionales" que tuvieron que llevarse a cabo, las técnicas e instrumentos utilizados -y su justificación-, las fuentes de información consultadas -bibliográficas, principales y accesorias- con las que se debió trabajar, etc. ${ }^{11}$

Quizás podría asociarse la formulación de una adecuada estrategia de investigación exploratoria con ciertas "prácticas de ensayo" de determinados modos de abordaje (teóricos y metodológicos) del problema propuesto. Dichos modos permitirían realizar, a manera de ejemplo y entre otras actividades posibles, las siguientes: (1) desagregar (pre)hipótesis preliminares en términos de variables e indicadores ("valores" que podrán identificarse y "mediciones" que podrán operacionalizarse) ${ }^{12}$; (2) elegir las técnicas (documental, estadística, análisis de contenido) e instrumentos (estudio de fuentes o registros, observación participante, entrevista, encuesta) más idóneos para indagar las variables propuestas; (3) establecer correspondencias entre unas y otros; (4) justificarlos, en términos de confiabilidad (grado de aplicación) y de validez (efectiva relación con lo que se pretende analizar o medir); etc.

realización de tareas exploratorias, y su aporte a la generación de hipótesis); las segundas, con el contexto de justificación científica (de resultados obtenidos y su correspondiente significatividad).

No obstante su diversidad paradigmática y sus diferencias estratégicas (para la recolección y el análisis de los datos obtenidos, por ejemplo, ya que implican marcos teóricos y metodológicos distintos), es posible "combinarlas" en una práctica investigativa concreta, toda vez que una adecuada integración de las mismas suele posibilitar una mejor descripción y comprensión de los fenómenos estudiados.

En síntesis: no se trata de subordinar unas metodologías a otras, sino de articularlas o combinarlas en un mismo proyecto o tratamiento de caso, en las investigaciones sociales, ya quela investigación cualitativa puede ayudar, por ejemplo, a "interpretar, iluminar, ilustrar y cualificar las relaciones determinadas estadísticamente".

${ }^{11}$ En función de los objetivos perseguidos, en esta instancia del trabajo se pueden precisar también los temas, subtemas, etc. que demandaron ser abordados para dar cuenta de dichos propósitos; y en relación con cada uno (de los temas, subtemas, etc.), sería conveniente precisar luego las actividades que fue necesario realizar.

${ }^{12}$ Consideremos un ejemplo: la existencia de numerosas becas anuales de posgrado e investigación puede ser un "indicador" de explícitas políticas de fomento científico; y esta variable, junto con muchas otras (diversificación de proyectos por áreas disciplinares, intensificación de la actividad de posgrado, etc.), permitiría a su vez abordar el tratamiento de las relaciones entre enseñanza y desarrollo científico en una determinada institución universitaria. 


\subsection{ANÁLISIS}

De las diferentes prácticas descriptivas, comprensivas e interpretativas ${ }^{13}$ y que, al menos en la propuesta que ejemplificaremos a continuación, constituyen los distintos casos y/o recorridos fragmentarios de lectura textual de un determinado corpus. Implican, en realidad, distintos modos posibles de responder a las preguntas referidas a cómo puede ser investigado el problema en cuestión/ el objeto de estudio previamente construido.

\section{Tres observaciones generales:}

> Si no fueron incorporadas en el apartado anterior, cada una de las lecturas practicadas debiera incluir, por lo menos: (a) una breve descripción de las alternativas metodológicas seleccionadas (de entre las disponibles); (b) una somera definición o construcción del marco teórico ${ }^{14}$ que las sustenta, con el agregado (explicitación o explanación) de sus supuestos e implícitos; y (c) otras referencias al diseño de la práctica de lectura que se consideren pertinentes.

> Debido a su propia índole discursiva, la redacción de esta parte "especial" del informe podrá caracterizarse por la presencia de un lenguaje "connotativo", de nuevos recursos expresivos, retóricos o estilísticos que la diferencian de los apartados anteriores, particularmente si se trata de lecturas referidas a textos artísticos, literarios, etc. $>$ Para el caso de resolverse un planteamiento de diferentes exploraciones analíticointerpretativas, tal vez sería conveniente redistribuir las diferentes prácticas de lectura propuestas según su correspondiente modalidad de abordaje.

\footnotetext{
${ }^{13}$ El presente formulario propone distribuir la información concerniente a las prácticas de lectura textual desarrolladas en el informe, entre este apartado y los correspondientes al rubro Anexos (ver más abajo). Dicho en otros términos: como el enunciado de los resultados de la exploración practicada debe caracterizarse por un mínimo de claridad, precisión y objetividad escritural, es conveniente que tales ítems del "desarrollo del informe" remitan a la elaboración posterior de diferentes gráficos, tablas, esquemas o sinopsis que, insertos más adelante en el texto, facilitan y permiten retomar así los aspectos analizados, sin "interrumpir" en este momento de la exposición su ordenamiento conceptual básico.

${ }^{14}$ Las interrelaciones posibles de ser establecidas entre teoría, método y práctica de investigación suelen suscitar innumerables discusiones y debates en la comunidad científica, aunque "toda teoría ha de basarse en hallazgos empíricos para no quedar en una mera especulación ideológica [y] toda investigación debe partir de una teoría para [así] adquirir una mayor relevancia" (Cea d'Ancona, 1996).
} 
Por ejemplo:

\subsubsection{RECORRIDOS DE LECTURA}

Prácticas relativamente lineales o unidireccionales de lectura, en el sentido de "aplicaciones", transferencias o transposiciones de puntos de vista o posiciones únicas -exclusivas/excluyentes- en cuanto a sus marcos de referencia teóricometodológicos.

\subsubsection{LECTURAS POLIGONALES}

Contrariamente a los anteriores, se harán converger aquí distintos datos, teorías y/o metodologías -intra o intermétodos- en el abordaje simultáneo de un mismo aspecto o situación ${ }^{15}$.

\subsubsection{OTROS MODOS DE LECTURA}

Serán incorporados aquellos que hayan sido desarrollados durante la investigación.

${ }^{15}$ Asocio la noción de poligonalidad con el concepto de "triangulación", en este caso aplicado a la investigación en ciencias sociales: esto es, como sostiene Cea d'Ancona, "la utilización de múltiples puntos de referencia para localizar la posición exacta de un objeto en el espacio". Procedente en realidad de otros ámbitos del conocimiento (la topografía, la navegación, la logística castrense), la necesidad de triangular o articular distintos métodos en una misma investigación humanística, social, artística, etc., recién empezó a sostenerse en las últimas décadas, una vez considerada la conveniencia de poder combinar, por ejemplo, los resultados de una encuesta con las conclusiones de un trabajo de campo, u otras técnicas concretas de recolección de información como la entrevista o la observación participante. Más difundida y (en general) considerada más confiable que la triangulación intramétodo (es decir, el empleo de diferentes técnicas o instrumentos de una misma metodología, para el cumplimiento de un mismo objetivo), la triangulación entre métodos consiste en la combinación (cruzamiento) de metodologías no similares, aunque relativamente 'compatibles', para enriquecer indagaciones, mediciones, etc. aplicadas a una misma unidad de análisis. 


\section{CONCLUSIONES}

\subsection{SÍNTESIS}

Una vez descriptos, analizados, interpretados, etc. los lineamientos teóricoprácticos de los recorridos "fragmentarios" de lectura propuestos, se sincretizarán en el informe las operaciones desarrolladas y sus principales resultados o "hallazgos" investigativos más relevantes.

\subsection{EVALUACIÓN}

Se valorizarán a continuación los resultados obtenidos, cotejándolos con las (pre)hipótesis iniciales y los objetivos del estudio exploratorio, y procurando enfatizar los efectos de esta reflexión en tanto posibles respuestas obtenidas en relación con los problemas planteados en la introducción del informe.

\subsection{RECAPITULACIÓN}

Se retoman también aspectos ya señalados, para recapitular acerca de los logros más significativos de la experiencia, programar los pasos siguientes de la investigación en curso y/o anticipar indagaciones futuras que permitan dar respuesta a cuestiones aún pendientes de resolución.

\section{ANEXOS}

Quizás convendría que los diferentes anexos, apéndices, suplementos, diagramas, esquemas, gráficos, tablas, sinopsis, etc. que se deseen incorporar al trabajo, con el propósito de facilitar o complementar la lectura e interpretación del informe y sus resultados (aunque sin interrumpir la "continuidad" de la exposición): (1) se agreguen, en distintas páginas, al final del cuerpo del informe; (2) se identifiquen con números romanos (ej. Gráfico I, Gráfico II...) más una breve leyenda o (sub)título que los caracterice; y (3) se anticipen o anuncien durante el desarrollo del informe, preferentemente a través de referencias o notas al pie (por ej. ver o cf. Esquema II, p. 10; etc). 


\section{BIBLIOGRAFÍA ${ }^{16}$}

Se sugiere que, en cada uno de los ítems mencionados a continuación, se citen las referencias bibliográficas siguiendo la ordenación alfabética, por apellido de autor(a)(es), y en caso de repetirse la autoría, por año de edición:

\subsection{CORPUS TEXTUAL ANALítico}

Se agregará, en cada caso, toda precisión complementaria relacionada con cuestiones de autoría: ej. autor del prólogo o estudio preliminar, equipo de producción del objeto o corpus analizado, etc.)

${ }^{16}$ Si bien existen diferentes técnicas/normas de notación bibliográfica, para la elaboración de estos informes de investigación propongo adoptar las siguientes:

> Para las referencias de libros: Apellido e inicial del nombre del autor [ed. o comp., si correspondiera] (año de la 1 a ed.) Título del libro (en cursiva/y si se diera el caso: "título del capítulo o del artículo", en...). Traductor. Ciudad: Editorial. También, si correspondiera: número y año de la edición (manejada para la redacción del informe). Ejemplos: - Gorlier, J.C. (2008) ¿Confiar en el relato? Narración, comunidad, disidencia. Mar del Plata: EUDEM

- Greimas, A.J. y J. Courtés (1979) "Prefacio" y "Nota a la edición en lengua española", en Semiótica. Diccionario razonado de la teoría del lenguaje. Trad. E. Ballón Aguirre y H. Campodónico Carrión. Madrid: Gredos. 2a ed., 1991. pp. 7-19.

- Jitrik, N. [comp] (1990) Irrupción del discurso. Interdisciplina y transdisciplinariedad. México: Universidad Autónoma. - Pavis, P. (1980) Diccionario del teatro. Dramaturgia, estética, semiología. Trad. F. de Toro. Barcelona: Paidós. 2a ed., 1998. - _- (2000) El análisis de los espectáculos. Teatro, mimo, danza, cine. Barcelona: Paidós

- Siles, J. (2008) "La traducción poética como proceso creativo", en Tortosa, V. [ed] Re-escrituras de lo global. Traducción e interculturalidad. Madrid: Biblioteca Nueva. pp. 487-501.

$>$ En las REFERENCIAS DE REVISTAS Y OTRAS PUBLICACIONES PERIÓDICAS: se agregarán al título del artículo citado, el nombre y número/volumen de la publicación (en cursiva), fecha y páginas del artículo. Ejemplos:

- Alonso, F. y otros (2007) "La entrevista: testimonios, trayectorias, prácticas". De signos y sentidos. Cuadernos del proyecto, 7. Santa Fe: Ediciones UNL. Agosto 2008. pp. 23-37.

- Caudana, C. (2001) "La semiosis didáctica (constituciones, formatos y soportes)". Texturas. Estudios interdisciplinarios sobre el discurso, 2. Santa Fe: Ediciones UNL. Diciembre 2002. pp. 33-59. 


\title{
6.2. REFERENCIAS BIBLIOGRÁFICAS
}

\author{
Sólo se incorporarán aquí los textos bibliográficos explícitamente mencionados \\ y/o citados en el informe.
}

\subsection{BIBLIOGRAFÍA GENERAL}

Sólo se incluirán, en este apartado, otros textos efectivamente consultados para la elaboración del trabajo, aunque no hubieran sido citados en el cuerpo del informe.

Se sugiere distinguir, dentro de las referencias bibliográficas consultadas, entre los textos (a) de apoyo teórico y metodológico, (b) sobre temas de interés o áreas afines al informe y (c) otros textos consultados.

- Garasino, A. (1965) "Radiografía militar del golpe". Confirmado, 6. 23 de junio de 1965. pp. 60-64.

$>$ Respecto de las referencias obtenidas en TEXTOS ELECTRÓNICOS, BASES DE DATOS, PROGRAMAS INFORMÁTICOS, PUBLICACIONES SERIADAS ELECTRÓNICAS se sugiere adoptar la siguiente notación: Responsable (y año) de la contribución, "Título" y [soporte]. Titulo del texto, documento fuente o publicación seriada. <sitio de la publicación > y [fecha de consulta]. Ejemplos:

- Svenonius, E. (2007) "References vs. added entries" [cd-rom]. En: Authority Control in the 21st Century. Dublin, Ohio: OCLC. <http://www.oclc.org/oclc/man/authconf/svenoniu.htm> [Consulta: 27 abril 2009].

- Willet, P. (2009) "The Victorian Women Writers Project [on line]: the library as a creator and publisher of electronic texts". The public-access computer systems review, II - 6. <http://info.libuh.edu/pr/v7/n6/will7n6.htm> [Consulta: 27 abril 2009].

> OTRAS CONSIDERACIONES: (1) A continuación de las citas bibliográficas (textuales -entrecomilladas- o indirectas -idea/concepto, no textual) y otras referencias indicadas en el cuerpo del informe, consignar por ej.: [Greimas, 1979: 135-6], es decir, apellido del autor, año de edición (ambos datos remiten a 5. Bibliografía) y página(s) correspondiente(s) a la cita o idea/concepto. (2) Si el autor tuviera más de una publicación en el mismo año: mencionar por ej. "En estudios anteriores [Greimas, 1979a: 15; 1979b: 37] el mismo autor sostuvo que..." (3) Trabajos de varios autores: la primera vez se citan todos los apellidos [Greimas, Courtés y Fontanille] y, en las citas posteriores: [Greimas y otros]. (4) Para el caso de comentarios e investigaciones mencionadas en otros trabajos: "Greimas [1979, citado por Fontanille, 1994: 35] propuso que..." (5) Si no se consigue identificar la fecha de publicación del texto, emplear la abreviatura s.f.; si no es posible identificar al autor, iniciar la referencia con el título del documento o artículo; si el trabajo es anónimo, iniciar la referencia con la palabra Anónimo (en el lugar del autor). 


\begin{tabular}{|l|l|}
\hline ESQUEMA DE CONTENIDO DEL INFORME & EXTENSIONES (tentativas) ${ }^{17}$ \\
\hline 1. PARATEXTOS & dos páginas \\
1.1. Portada & \\
1.2. Índice o sumario & \\
\hline 2. INTRODUCCIÓN & dos / cuatro páginas \\
2.1. Preliminares & $150 / 300$ palabras \\
2.2. Argumentos & $500 / 1000$ \\
2.3. Contexto & $300 / 800$ \\
2.4. Agregados & [hasta 300$]$ \\
\hline 3. DESARROLLO & cuatro / diez páginas \\
3.1. Estrategias & $400 / 800$ palabras \\
3.2. Análisis & \\
3.2.1. Recorridos de lectura & $500 / 1000$ palabras \\
3.2.2. Lecturas poligonales & para cada lectura \\
3.2.3. Otros modos de lectura & o abordaje practicado \\
\hline 4. CONCLUSIONES & una / dos páginas \\
4.1. Síntesis & $200 / 500$ \\
4.2. Evaluación & palabras referidas \\
4.3. Recapitulación & a cada aspecto \\
\hline 5. ANEXOS (apéndices, diagramas, esquemas, etc.) & - - - - - \\
\hline 6. BIBLIOGRAFíA & -- - - - \\
6.1. Corpus textual analítico & \\
6.2. Referencias bibliográficas \\
6.3. Bibliografía general \\
6.3.1. De apoyo teórico y metodológico \\
6.3.2. Temas de interés o áreas afines \\
6.3.3. Otros textos consultados & \\
\hline
\end{tabular}

${ }^{17}$ Las extensiones tentativas/sugeridas se proponen o calculan sobre la siguiente base/promedio: (a) fuente arial/ tamaños 11 (texto principal), 10 (citas), 9 (notas); (b) interlineado exacto 15 (texto principal) y sencillo (citas y notas); (c) márgenes sup 3, inf 2, izq 3,5, der 1,5; (d) sangrías $1+1$ (citas), especial 1 ra línea 0,5. 